\title{
Author Correction: Solid-state nanopore sensors
}

\author{
Liang Xue (D), Hirohito Yamazaki, Ren Ren, Meni Wanunu (D), Aleksandar P. Ivanov (D) and Joshua B. Edel(D)
}

Nature Reviews Materials (2020) https://doi.org/10.1038/s41578-020-0229-6 Published online 21 September 2020

The originally published article contained some wrongly numbered references. In the first paragraph of the "Atomically thin 2D membranes" section, the original text read "There are two dominant methods used for fabricating free-standing 2D membranes: transfer of a 2D material prepared by exfoliation ${ }^{110,111}$ or CVD ${ }^{80,98,99,103,104,105,106,109}$ on a membrane with a relatively large sub-micrometre aperture (Fig. $1 \mathrm{~h}$ ); or growth of the 2D material directly on the aperture using CVD ${ }^{100,108,112}$ (Fig. 1i). The first method is more common, as the procedures for material transfer are well established and the system is easy to inspect optically ${ }^{113}$. Direct CVD grown on the aperture is scalable to whole wafers ${ }^{112}$, but the process is challenging to control." The correct references in this paragraph are "There are two dominant methods used for fabricating free-standing 2D membranes: transfer of a 2D material prepared by exfoliation ${ }^{110,111}$ or $\mathrm{CVD}^{80,98,99,103,104,105,106,109,112}$ on a membrane with a relatively large sub-micrometre aperture (Fig. 1h); or growth of the 2D material directly on the aperture using CVD ${ }^{100,108}$ (Fig. 1i). The first method is more common, as the procedures for material transfer are well established and the system is easy to inspect optically ${ }^{113}$. Direct CVD grown on the aperture is scalable to whole wafers ${ }^{108}$, but the process is challenging to control."

This has been corrected both in the PDF and in the HTML versions of the article.

https://doi.org/10.1038/s41578-020-00256-x I Published online 1 October 2020

(c) Springer Nature Limited 2020 